\title{
EXPLORING THE EFFECTS OF STRUCTURAL TRANSPARENCY AND EXPLORATORY GUIDANCE IN SIMULATION-BASED LEARNING ENVIRONMENTS
}

\author{
Carlos Capelo ${ }^{1}$ and Ana Lorga ${ }^{2}$ \\ ${ }^{1}$ Universidade Europeia, Estrada da Correia, 53, 1500-210 Lisboa-Portugal \\ ${ }^{2}$ Universidade Lusófona de Humanidades e Tecnologias, Campo Grande, 376, 1749-024 Lisboa-Portugal
}

\begin{abstract}
Simulation-based learning environments are used extensively to support learning on complex business systems. Nevertheless, there exist studies that identify problems and limitations due to cognitive processing difficulties. Particularly, previous research addressed some aspects of model transparency and instructional strategy and produced inconclusive results about their impact on learning effectiveness. This study investigates the learning effects of using transparent simulations (that is, showing users the internal structure of models), and exploratory guidance (that is, guiding learners so they are able to explore the simulation by themselves, supported by specific cognitive aids). We present a set of hypotheses about the influence of the degree of simulator transparency and the degree of exploratory guidance on participants' model comprehension which is assessed in terms of mental model structure and behaviour similarities. A test based on a simulation experiment with a system dynamics model, representing a supply chain system, was performed. Participants are required to use the simulator to investigate on some issues related to the bullwhip effect and other supply chain coordination concepts. Participants provided with the more transparent strategy and offered the more exploratory guidance demonstrated better understanding of the structure and behaviour of the underlying model. However, our results suggest that while exploratory guidance is a beneficial method for both model structure and behaviour understanding, making solely the model transparent is more limited in its effect.
\end{abstract}

\section{KEYWORDS}

Management Education, Simulation-Based Learning, System Dynamics, Model Transparency, Instructional Guidance, Supply Chain Management, Bullwhip Effect

\section{INTRODUCTION}

\subsection{Simulation-Based Learning in Management Education}

Research has suggested that the use of SBLEs frequently induces active learner behaviour, scientific reasoning and constructive learning processes (de Jong and van Joolingen 1998). Particularly, studies have shown that (SBL) with system dynamics (SD) can support and enhance learning. SD is a scientific approach for (computer-based) modelling and simulation developed to facilitate our understanding and management of complex, dynamic systems (Davidsen and Spector, 2015). The models are expressed graphically so as to facilitate effective description about the systems they intend to represent.

From a mental models perspective, students can use the simulation program to support the construction and improvement of their mental models. They form an initial mental model and develop into a target conceptual model (the same one underlying the simulation model). Moreover, SBL facilitates improving the learner's mental models by engaging in inquiry that is otherwise impractical or even impossible.

Simulators are promising tools for teaching in the management domain. Studies have shown that students perceive simulation as a more effective method than text-based case study and lecture (Farashahi and Tajeddin, 2018). However, evidence regarding the impact of their utilization remains limited (Lean at al., 2015). Therefore, a need exists for more research addressing how the learning potential of such environments might be enhanced (Davidsen and Spector, 2015). According to the literature, the effectiveness of SBLEs depends on many factors related to the learning context. The present study focusses on two aspects: simulator transparency and instructional strategy. 


\subsection{Simulator Transparency and Instructional Guidance}

Simulator transparency refers to what extent the structure of the underlying computational model is shown to students using the simulation. In black-box model simulations, students can explore a system's behaviour, but the underlying computational models remain hidden and can only be inferred by what appears on the screen. Studies have suggested that this type of "black-box" situation could lead students to form wrong mental models, interfering with proper learning. "Glass-box model" or "transparent" simulations have alternatively been proposed to obviate the above-described problems, as their relations among variables are accessible by the students. Transparent simulators have been used in SD learning environments, which provide their stocks-and-flows diagram (SFD) detailing the causal structure of the underlying system. By using this approach, students may trace the cause and effect structure and understand the relationships between structure and behaviour (Milrad et al., 2003), and they have the potential to understand even counterintuitive system behaviours (Groesser, 2012).

Research shows that structural knowledge provided by transparent simulations has the potential to improve leaning and task performance (Größler et al., 2000; Qudrat-Ullah, 2007). Kopainsky and Alessi (2015) found that participants provided with the more transparent strategy demonstrated better understanding of the underlying model, but their performance, however, was the equivalent to those in the less transparent condition. The implication of previous study is that the students must not only identify the structure of the simulator model, but will also have to recognize the relationship between structure and behaviour (Davidsen and Spector, 2015).

Instructional guidance refers to the support provided to students during simulation in the form of questions, hints, procedures, steps, or materials. The associated issue is about how much and what type of instructional guidance needs to be offered in order to optimize the learning potential of simulations. In SBL, direct instruction occurs when detailed instructions are given, and students must learn the underlying concepts through a sequence of programmed steps. In inquiry learning method, general questions or learning goals are presented, and students are free to explore the SBLE. Studies have found that students achieve deeper understanding of subject matter when developing scientific reasoning (Clement, 2008), as they formulate hypotheses, define actions and scenarios, conduct experiments, interpret simulation results, and draw conclusions. However, research on inquiry learning with computer simulations (for example: de Jong, 2006) found evidences that students operating in complex simulation environments generally have difficulty in all phases of the inquiry process. Cognitive load theory (Sweller et al., 2011) provides an explanation for those problems. In a simulation task, the complexity of the model may exceed the working memory limits of participants. To obviate these "cognitive load" problems and increase the effectiveness of simulation, de Jong (2006) suggests that instructional method be integrated with "cognitive tools" aimed at guiding and supporting the students' activities. Van Borkulo et al. (2012) argue that, to enhance model-based learning, students need to be walked through each element of the model (approach known as model progression), allowing participants to incrementally build their mental models. Mulder et al. (2015) examined how model progression and worked examples can promote inquiry learning and found positive effects due to both increasing model complexity and providing worked examples.

\section{RESEARCH HYPOTHESES}

This research focuses on the issue of which model transparency and instructional guidance conditions are the most suited for optimizing the learning potential of simulation for teaching the bullwhip effect and SCC, leading to enhanced student understanding of their main concepts. The analysis of the students' understanding of the simulation model considers two components: the comprehension of model structure and the comprehension of model behaviour. The expected relations and hypotheses are based on the following variables:

(i) Level of Model Transparency (LMT) - this variable represents the transparency level of the simulator. LMT indicates to what extent students have access to the variables and relations included in the model.

(ii) Level of Exploratory Guidance (LEG) - this variable represents the exploratory level of the instructional method used to guide students throughout the simulation task. LEG indicates to what extent students may choose methods and activities to explore the SBLE. 
(iii) Comprehension of the model structure (CMS) - this variable indicates to what extent students understand the structure of the simulation model. CMS measures the similarity between the structure of the external representation of the students' mental models and the structure of the simulation model.

(iv) Comprehension of the model behaviour (CMB) - this variable indicates to what extent students are able to understand and infer the dynamical behaviour of the SCC simulator. CMB measures the similarity between the student's expectation on model behaviour (inference of student's mental models) and the actual behaviour of the simulation model.

The following hypotheses are defined: H1- LMT positively influences CMS, H2- LMT positively influences CMB, H3- LEG positively influences CMS, H4- LEG positively influences CMB, and H5- CMS positively influences CMB.

\section{METHOD}

\subsection{Simulator, Participants, Procedure, and Facilitation}

The SD model incorporated in the simulator represents a supply chain of a beer distribution system. It is based on the famous board game (the beer game) first developed in the 1960s at the Massachusetts Institute of Technology. The simulator interface includes three screens. The first screen is separated into three sections: (i) one section allows participants to adjust simulation parameters, (ii) a second section include three graphics that present the historical behaviour over time for inventory, supply flows, and order backlog for each of the supply chain stages, and (iii) a third section with a table presenting performance measures. A second screen provides a description of each of the variables in use. A third screen, when available, shows the SFD of the simulator model.

The participants define the experiment scenario, adjust the parameters accordingly and run the supply chain simulator for a period of six months. Then, they observe the graphics to analyse the behaviour over time and appreciate the overall performance indicators.

In order to test the model of hypotheses, a $2 \times 2$ experimental design with four treatment groups was defined: two groups (A and C) of participants who interact with an opaque simulator (low LMT), two groups (B and D) who use a transparent simulator (high LMT), two groups (A and B) who receive step by step instructions (low LEG), and two groups (C and D) who receive exploratory guidance (high LEG).

This research was conducted at Universidade Europeia in Lisbon. The experiment involved four classes of supply chain management courses (with 93 students in total). Each one of the four different experimental treatments was assigned randomly to one of the four classes. As participants from group A are submitted to the basic conditions (low LMT and low LEG), the present quasi-experiment design considers groups $\mathrm{B}, \mathrm{C}$, and D as experimental groups, while group A assumes the role of control group.

All the participants, from both experimental and control groups, were familiar with the concepts included in the simulator, as they attended lecture classes on supply chain coordination and bullwhip effect. The experiment was carried out individually in class with a participant per computer. All students were given a full experimental guide including: presentation of the simulator, objectives, instructions for accessing, starting, and running the simulator, instructions for performing the simulation task, SFD of the simulator model (only for treatment groups B and D), and sheets for recording scenarios and results.

In the simulation experiment, the participants were asked to investigate the impact of some factors on supply chain performance. The following five factors were considered: demand irregularity, demand information sharing, supply line control, order fraction, and inventory cover. Past research found that students operating in complex simulation environments generally have considerable difficulty in all phases of the inquiry process (de Jong 2006). To obviate these problems, previous studies (van Borkulo et al.,2012; Elsawah et al., 2017; Mulder et al., 2015) suggest that participants need to be guided through the simulation model, gradually increasing the task complexity (model progression). And according to Mulder et al. (2015), complementing model progression with worked examples enhances students' inquiry performance and learning. Consequently, the students from groups $\mathrm{C}$ and $\mathrm{D}$ (submitted to exploratory guidance) address the five factors sequentially and one at a time. And they are also guided to firstly analyse performance effects caused by the factor being addressed and then to look at potential interactions with other factors previously 
analysed. Additionally, the students (from groups $\mathrm{C}$ and D) receive a demonstration on how to investigate the supply chain effects of the first factor (demand irregularity) by defining hypotheses and scenarios, adjusting the parameters, running the simulator, and interpreting the results.

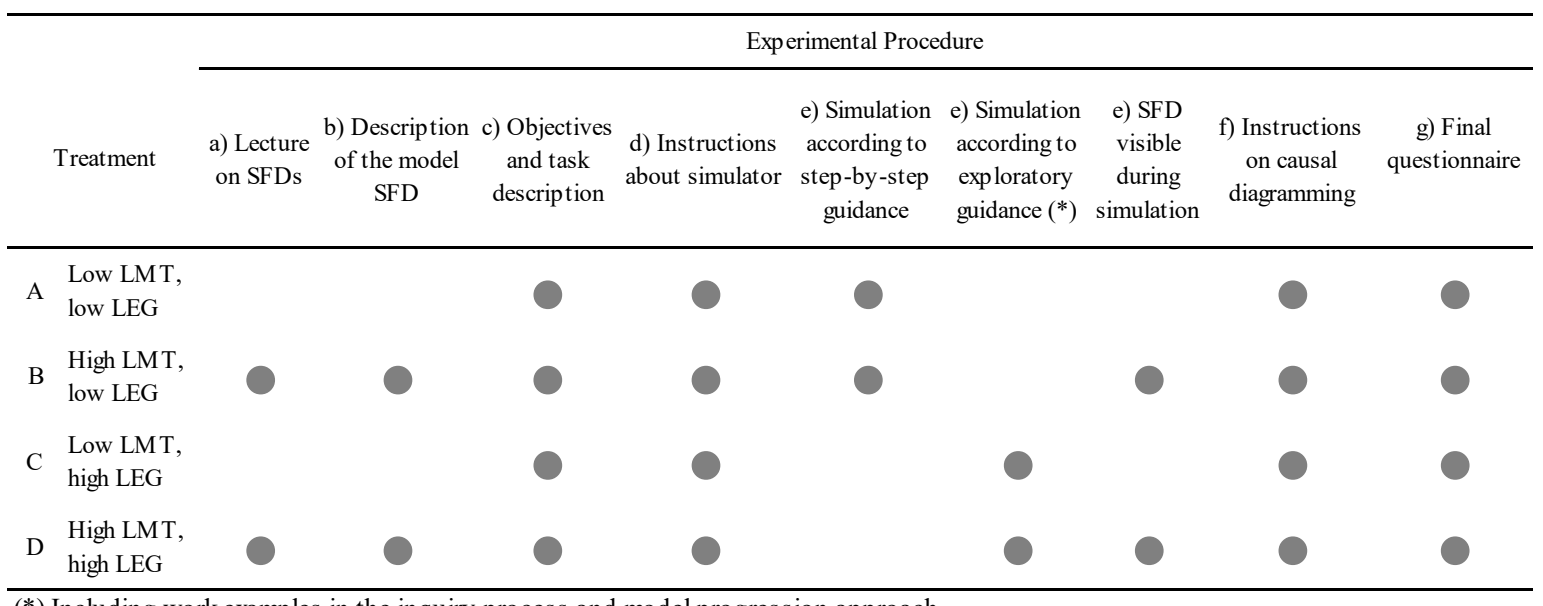

(*) Including work examples in the inquiry process and model progression approach

Figure 1. Experimental procedure

The experiment procedure involved three sessions and had the following steps (figure 1).

Session 1: This session involved only the participants from experimental groups B and D. (a) The students received a lecture on SFDs, so that they were able to read and interpret the SFD representing the simulator model. Then, (b) the instructor described the SFD to the students in a form of a guided tour.

Session 2: In this session, the students performed the simulation task. (c) The students read the introduction with the overall description and the objectives of the simulation task. (d) The participants read the instructions for accessing, starting and running the simulator. Some simulation runs were conducted to familiarise participants with the game interfaces and commands. (e) The participants performed the simulation task, following the instructions included in the experimental guide. Students from experimental groups $\mathrm{A}$ and $\mathrm{B}$ adjusted the parameters and run the simulator according to step-by-step instructions that guide them to discover the performance effects of those parameters. Students from experimental groups $\mathrm{C}$ and $\mathrm{D}$ were free to explore the simulator. They analysed the questions to be addressed, defined hypotheses and scenarios, adjusted the selected parameters, run the simulator, interpreted the results, defined new hypotheses scenarios, and repeated the process. As mentioned before, the students from groups C and D were guided through work examples on how to perform this inquiry process and according to model progression approach. During the simulation task, the participants from experimental groups B and D were encouraged to read the SFD. The students were also instructed to ask for support if they found any difficulties in reading the SFD (groups B and D) or in any stage of the inquiry process (groups C and D).

Session 3: (f) Firstly, the students received instruction in how to read and draw a causal-loop diagram (CLD). (g) Then, they individually answered a questionnaire to measure learning on the simulator model, which consists of two parts. In the first part, they were asked to complete a CLD representing the simulator model. Students had to fill in the empty boxes writing the corresponding concepts, which they selected from a list of eleven missing concepts. They also had to identify eight missing links between the concepts and draw the corresponding arrows and signs that indicated the cause-and-effect relationships. In the second part the students responded to twenty multiple-choice questions related to the behaviour of the model. No pre-test was given (before the simulation task) because the topic tested (simulator model) was new material for all the students. And they did not previously know about the questionnaire that they would be required to answer.

\subsection{Research Variables}

This section summarises the use of the variables that were defined in the research model. 
Level of Model Transparency (LMT) - This variable features two degrees. In the low degree (low LMT), the students perform the simulation task without accessing the SFD of the simulator model (treatment groups A and C). In the high degree (high LMT), the students, during simulation task, have access to the SFD representing the simulator model (treatment groups B and D).

Level of Exploratory Guidance (LEG) - This variable features two degrees. In the low degree (low LEG - treatment groups A and B), the students perform the simulation task by strictly following the "step-by-step" instructions provided. For each issue being addressed, they replace the parameters with the values given, run the simulator and observe the results. The students are not encouraged to reason scientifically. In the high degree (high LEG - treatment groups C and D), the students are free to explore the simulator, performing all the simulation runs they need. This experiment condition is designed in order to foster scientific reasoning and promote inquiry learning with appropriate aids. They are asked to investigate the supply chain performance effects of the selected factors. The students reflect on each issue, formulate some hypotheses about model behaviour, and define experimental scenarios in order to test those hypotheses (including the parameters to be adjusted and corresponding values). They run the simulator, interpret the results, test the hypotheses, attempt to explain model behaviour, and determine further steps before the cycle repeats.

Comprehension of the model structure (CMS) - this variable measures the similarity between the structure of the external representation of the students' mental models and the structure of the simulation model. As the structure of the simulation model is known by the researchers in advance, it can be compared with the participants' mental model in order to evaluate how that elicited mental model matched the simulated reality. As described in previous section, the students were asked to complete a causal-loop diagram representing the simulator model by inserting some missing concepts (eleven) and links (eight). This diagram represented the elicited structure of the students' mental model. Each concept or link answered was worth one point. Thus, CMS ranges from 0 to 19 .

Comprehension of the model behaviour (CMB) - this variable measures the similarity between the student's expectation on model behaviour (inference of student's mental models) and the actual behaviour of the simulation model. In the second part of the final questionnaire, the students responded to multiple-answer questions related to the dynamical behaviour of the model (20 questions). Each question was worth one point. Thus, CMB ranges from 0 to 20 .

\section{RESULTS AND DISCUSSION}

Table 1 presents the mean values, standard deviations, and sample sizes for the dependent variables CMS and $\mathrm{CMB}$ corresponding to the four experimental treatments. Table 2 displays the results of an independent-samples t-test of significance for differences in means between pairs of treatment groups.

Table 1. Means and standard deviations for variables CMS (Comprehension of Model Structure) and CMB (Comprehension of Model Behaviour) for the four experimental groups

\begin{tabular}{|c|c|c|c|c|c|c|}
\hline \multirow[b]{2}{*}{ Treatment } & \multirow[b]{2}{*}{ Description } & \multirow[b]{2}{*}{$\mathrm{N}$} & \multicolumn{2}{|c|}{$\begin{array}{l}\text { CMS - Comprehension of } \\
\text { Model Structure }\end{array}$} & \multicolumn{2}{|c|}{$\begin{array}{l}\text { CMB - Comprehension of } \\
\text { Model Behaviour }\end{array}$} \\
\hline & & & Mean & SD & Mean & SD \\
\hline $\mathrm{A} / \mathrm{CG}$ & Low LMT, low LEG & 24 & 6.167 & 2.761 & 5.833 & 1.659 \\
\hline B & High LMT, low LEG & 23 & 9.609 & 5.408 & 6.217 & 2.194 \\
\hline $\mathrm{C}$ & Low LMT, high LEG & 22 & 8.000 & 4.546 & 7.227 & 2.581 \\
\hline $\mathrm{D}$ & High LMT, high LEG & 24 & 14.292 & 4.379 & 8.667 & 2.929 \\
\hline
\end{tabular}

Variable definitions: LMT - Level of Model Transparency; LEG - Level of Exploratory Guidance 
Table 2. Independent-samples t-test of significance for differences in means between pairs of treatment groups

\begin{tabular}{|c|c|c|c|c|c|c|}
\hline \multirow[b]{2}{*}{ Pair } & \multicolumn{3}{|c|}{ CMS - Comprehension of Model Structure } & \multicolumn{3}{|c|}{ CMB - Comprehension of Model Behaviour } \\
\hline & Mean Difference & SD & $\begin{array}{c}\text { Significance } \\
\mathrm{p}\end{array}$ & Mean Difference & SD & $\begin{array}{c}\text { Significance } \\
\mathrm{p}\end{array}$ \\
\hline B-A & $3.442 * *$ & 1.261 & 0.010 & 0.384 & 0.569 & 0.504 \\
\hline $\mathrm{C}-\mathrm{A}$ & 1.833 & 1.121 & 0.111 & $1.394 * *$ & 0.646 & 0.038 \\
\hline D-A & $8.125 * * *$ & 1.057 & 0.000 & $2.833 * * *$ & 0.687 & 0.000 \\
\hline D-B & $4.683 * * *$ & 1.439 & 0.002 & $2.449 * * *$ & 0.753 & 0.002 \\
\hline $\mathrm{D}-\mathrm{C}$ & $6.292 * * *$ & 1.318 & 0.000 & $1.439 *$ & 0.813 & 0.083 \\
\hline
\end{tabular}

${ }^{* * *} \mathrm{p}<0.01,{ }^{* *} \mathrm{p}<0.05,{ }^{*} \mathrm{p}<0.1$

The lowest mean values for the variables CMS and CMB were found in participants from group A (control group), which were submitted to the basic conditions (opaque simulation and "step-by-step" guidance). This result may be explained by the lack of essential information concerning the structure of the simulator model. Additionally, the "step-by-step" guidance did not foster scientific reasoning and inquiry learning, and this may have led participants to misinterpret cause-and-effect relationships and model behaviour.

On average, the participants from group D (transparent simulation and exploratory guidance) exhibited the highest values for the variables CMS (mean=14.292) and CMB (mean=8.667). As shown in Table 2, the mean values of CMS and CMB for group D were significantly different from the equivalent values for group A (pair D-A; CMS mean difference $=8.125, \mathrm{p}<0.001$; $\mathrm{CMB}$ mean difference $=2.833, \mathrm{p}<0.001$ ). These results suggest that students learn more effectively on SCC concepts if the SBLE combines transparency and a higher degree of exploratory guidance. These processes combined gave participants from group D a significant cognitive aid that accelerated their learning about the relationships between structure and behaviour of the simulated system.

The students from group A and those from the group B were submitted to equivalent guidance conditions ("step-by-step" instructional guidance). The difference between these treatment groups is that while students from group A used an opaque simulator, students from group B interacted with a transparent simulator. As expected, the comparison between treatment groups A and B presented in Tables 1 and 2 demonstrates that simulator transparency did cause the participants to improve their CMS. The statistical testing presented in Table 2 (pair B-A) provides evidence that the average responses from those groups are significantly different $($ CMS mean difference $=3.442, \mathrm{p}=0.010)$. On the other hand, the comparison between group D (transparent simulation and exploratory guidance) and group $\mathrm{C}$ (opaque simulation and exploratory guidance) reveals an even stronger difference in means for CMS (mean difference $=6.292, \mathrm{p}<0.000$ ). Consequently, the variable LEG seems to positively moderate the impact of LMT on CMS. Students demonstrate on average an increasing comprehension of the model structure if they combine transparent simulation with a higher degree of exploratory guidance.

As shown in Table 2, it is clear that the difference in means for CMS between group C (opaque simulation and exploratory guidance) and group A (control group) is not significant (mean difference $=1.833$, $\mathrm{p}=0.111$ ). This means that when students are performing the opaque simulation, the mean value of CMS for those students submitted to exploratory guidance is not significantly different from the equivalent value for those submitted to "step-by-step" guidance. However, the difference in means for CMS between group D (transparent simulation and exploratory guidance) and group B (transparent simulator and "step-by-step" guidance) is very significant (mean difference $=4.683, \mathrm{p}=0.002$ ). Thus, the variable LMT seems to positively moderate the impact of LEG on CMS. These results suggest that by using an exploratory guidance solely, students do not learn more effectively about the model cause-and-effect relationships. In other words, even though students from group $\mathrm{C}$ apply a scientific approach (hypothesis formulation and testing) supported by specific cognitive aids, they are not more able to derive structure from behaviour.

There were very few differences between the means of the CMB for the treatment A (CMB mean $=5.833)$ and treatment $\mathrm{B}(\mathrm{CMB}$ mean $=6.217)$. The independent-samples t-test be-tween treatments $\mathrm{A}$ and $\mathrm{B}$ displayed in Table 2 (pair B-A) does not suggest that by increasing simulator transparency, the participants revealed on average a higher CMB. Although the CMB mean increased with the use of the transparent simulator, as presented in Table 2, it is not significantly different (CMB mean difference $=0.384$, $\mathrm{p}=0.504$ ). Therefore, the null hypothesis, that transparent simulations will yield the same results as opaque 
simulations in foster their comprehension of model behaviour, cannot be rejected. As students from group B were explained the stocks-and-flows diagram of the simulator model, they took advantage of that structural knowledge and were more able to identify and represent the key variables and the feedback processes into a causal-loop diagram. Nevertheless, however, these students (from group B) were not more successful in comprehending the model behaviour. These results seem to evidence a learning difficulty, frequently mentioned in systems dynamics literature (Davidsen and Spector, 2015), that is difficult to develop an understanding of how the behaviour of a complex system emerges from its underlying causal structure. On the other hand, the comparison between group D (transparent simulation and exploratory guidance) and group $\mathrm{C}$ (opaque simulation and exploratory guidance) indicates a positive difference in means for $\mathrm{CMB}$ (mean difference $=1.439, \mathrm{p}=0.083$ ). Even though that difference is not very significant, it seems to suggest that the variable LEG also positively moderates the impact of LMT on CMB. Students demonstrate on average an increasing comprehension of the model behaviour if they combine transparent simulation with a higher degree of exploratory guidance.

Table 3. Regression results for all independent variables. Regression CMB (2) is obtained through a stepwise procedure

\begin{tabular}{|c|c|c|c|c|c|c|}
\hline \multirow[b]{3}{*}{ Independent Variables } & \multicolumn{6}{|c|}{ Dependent Variables } \\
\hline & \multicolumn{2}{|c|}{$\begin{array}{l}\text { CMS - Comprehension of } \\
\text { Model Structure }\end{array}$} & \multicolumn{2}{|c|}{$\begin{array}{l}\text { CMB - Comprehension of } \\
\text { Model Behaviour (1) }\end{array}$} & \multicolumn{2}{|c|}{$\begin{array}{l}\text { CMB - Comprehension of } \\
\text { Model Behaviour (2) }\end{array}$} \\
\hline & $\begin{array}{l}\text { Standardized } \\
\text { Beta }\end{array}$ & Significance $\mathrm{p}$ & $\begin{array}{l}\text { Standardized } \\
\text { Beta }\end{array}$ & Significance $\mathrm{p}$ & $\begin{array}{l}\text { Standardized } \\
\text { Beta }\end{array}$ & Significance $\mathrm{p}$ \\
\hline LMT & $0.462 * * *$ & 0.000 & $0.175^{*}$ & 0.071 & & \\
\hline LEG & $0.312 * * *$ & 0.001 & $0.373 * * *$ & 0.000 & $0.221 * *$ & 0.016 \\
\hline CMS & & & & & $0.482 * * *$ & 0.000 \\
\hline Adjusted R2 & \multicolumn{2}{|c|}{0.305} & \multicolumn{2}{|c|}{0.156} & \multicolumn{2}{|c|}{0.336} \\
\hline
\end{tabular}

$* * * \mathrm{p}<0.01, * * \mathrm{p}<0.05, * \mathrm{p}<0.1$

As seen from Tables 1 and 2, there was a significant difference between the means of the CMB for group $\mathrm{C}$ (opaque simulation and exploratory guidance) and group $\mathrm{A}$ (mean difference $=1.394, \mathrm{p}=0.038$ ). Moreover, the difference for $\mathrm{CMB}$ between treatments $\mathrm{D}$ (transparent simulator and exploratory guidance) and $\mathrm{B}$ (transparent simulator and "step-by-step" guidance) displayed in Table 2 (pair D-B: mean difference $=2.449$, $\mathrm{p}=0.002$ ) indicates a stronger significant difference than that found between groups $\mathrm{C}$ and $\mathrm{A}$. Consequently, these results seem to suggest that by increasing the level of exploratory guidance, the students demonstrate on average a higher CMB. But they may even learn more effectively about the dynamics of the SCC system if they combine exploratory guidance with transparent simulations. Thus, the findings indicate that LMT also positively moderates the impact of LEG on CMB.

As presented in Table 3, regression analysis for CMS on the independent variables shows a highly significant effect for LMT $(\beta=0.462, p<0.001)$ and a significant effect for LEG $(\beta=0.312, p=0.001)$. Regression analysis of CMB (1) show a low effect for LMT $(\beta=0.175, p=0.071)$, which is not significant (at a 0.05 level), and a highly significant effect for LEG $(\beta=0.373, p<0.001)$. Regression analysis of CMB (2) on the most significant independent variables (including CMS) shows a strong effect for CMS $(\beta=0.482$, $\mathrm{p}<0.001)$ and a significant effect for LEG $(\beta=0.221, \mathrm{p}=0.016)$.

\section{CONCLUSION}

This study is based on an education experiment aimed at testing hypotheses about the impact of simulator transparency and instructional guidance on students' learning. The simulation experience involves a simulator based on SD, designed to foster students' understanding of the bullwhip effect and other SCC concepts. The results from regression analysis confirm four of the five hypotheses. The hypothesis H1 (the level of transparency of the SCC simulation model positively influences the level of comprehension of the model structure) is supported. This finding is consistent with some of the literature on learning from transparent models (Grobler at al., 2000, Kompainsky and Alessi, 2015). However, the hypothesis H2 (the level of transparency of the SCC simulation model positively influences the level of comprehension of the model behaviour) is not full supported, thus suggesting that by using a transparent simulator solely, students do not learn about the model behaviour any more effectively than they would otherwise. This conclusion is in 
line with a learning difficulty frequently mentioned in systems dynamics literature (Davidsen and Spector, 2015), that is difficult to develop an understanding of how the behaviour of a complex system emerges from its underlying causal structure.

The hypotheses H3 (the level of exploratory guidance of the instructional method positively influences the level of comprehension of the SCC model structure) and H4 (the level of exploratory guidance of the instructional method positively influences the level of comprehension of the SCC model behaviour) are supported. The findings evidence a strong impact of the exploratory instructional process on learning about the SCC concepts. Students achieve a deeper understanding of the model structure and behaviour when they have opportunity to explore the simulation themselves and receive appropriate facilitation, such as worked examples and support on scientific reasoning (hypothesis formulation and testing) and model progression (gradually increasing task complexity). These results reinforce two assumptions already articulated in previous research. (i) The idea of using simulations to foster scientific reasoning and inquiry learning as pointed out by Clement (2008). (ii) And the importance of worked examples and model progression to obviate students' difficulties in all phases of the inquiry process and enhance learning as evidenced, for example, by Elsawah et al., (2017) (model progression) and Mulder et al. (2015) (model progression and worked examples).

Finally, the hypothesis H5 (the level of comprehension of the model structure positively influences the level of comprehension of the model behaviour) is also supported. This hypothesis was derived from the research question of whether or not improved mental models of systems lead to better inference of the systems behaviour. This finding is consistent with previous studies on this topic (Capelo and Dias, 2009).

\section{REFERENCES}

Capelo, C., Dias, J. 2009. A Feedback Learning and Mental Models Perspective on Strategic Decision Making. Educational Technology Research \& Development, 57: 629-644.

Clement, J. J. (2008). Creative model construction in scientists and students. The role of imagery, analogy, and mental simulation. New York: Springer.

Davidsen, P., Spector, J. (2015). Critical Reflections on System Dynamics and Simulation/Gaming. Simulation \& Gaming, 46, 430-444.

Elsawah, S., McLucas, A., Mazanov, J. (2017). An empirical investigation into the learning effects of management flight simulators: A mental models approach, European Journal of Operational Research, 259 (1), 262-272.

Farashahi, M., Tajeddin, M. (2018). Effectiveness of teaching methods in business education: A comparison study on the learning outcomes of lectures, case studies and simulations. The International Journal of Management Education, 16, $131-142$

Größler, A., Maier, F. H., \& Milling, P. M. (2000). Enhancing learning capabilities by providing transparency in business simulators. Simulation \& Gaming: An International Journal, 31, 257-278.

Groesser, S. N. (2012). Model-Based Learning with System Dynamics. In N. Seel (Ed.), Encyclopedia of the sciences of learning, pp 2303-2307. New York: Springer.

de Jong, T., van Joolingen, W. R. (1998). Scientific discovery learning with computer sim-ulations of conceptual domains. Review of Educational Research, 68(2), 179-201.

de Jong, T. (2006). Technological advances in inquiry learning. Science, 312, 532-533.

Kopainsky, B., Alessi, S. M. (2015). Effects of Structural Transparency in System Dynamics Simulators on Performance and Understanding. Systems, 3, 152-176.

Lean, J., Moizer, J., Warren, M. (2015). The use and impact of simulations in management education. The International Journal of Management Education, 13, 349.

Milrad, M., Spector, J. M., \& Davidsen, P. I. (2003). Model facilitated learning. In S. Naidu (Ed.), Learning and teaching with technology: Principles and practices, pp 13-27. London, England: Kogan Page.

Mulder, Y. G., Lazonder, A. W., de Jong, T. (2015). Simulation-based inquiry learning and computer modeling: Pitfalls and potentials. Simulation \& Gaming, 46, 322-347.

Qudrat-Ullah, H. (2007). Debriefing can reduce misperceptions of feedback: The case of renewable resource management. Simulation \& Gaming: An International Journal, 38, 382-397.

Sweller, J., Ayres, P., \& Kalyuga, S. (2011). Cognitive load theory. New York: Springer.

van Borkulo, S., van Joolingen, W., Savelsbergh, E., Jong, T. (2012). What can be learned from computer modeling? Comparing expository and modeling approaches to teaching dynamic systems behavior. Journal of Science Education and Technology, 21 (2), 267-275 2012. 